\title{
A High-Efficient Finite Difference Method for Flexible Manipulator with Boundary Feedback Control
}

\author{
Fushou Liu ${ }^{1}$ and Dongping Jin $^{2}$ \\ ${ }^{1}$ College of Civil Engineering, Nanjing Forestry University, 210037 Nanjing, China \\ ${ }^{2}$ State Key Laboratory of Mechanics and Control of Mechanical Structures, Nanjing University of Aeronautics and Astronautics, \\ 210016 Nanjing, China
}

Correspondence should be addressed to Dongping Jin; jindp@nuaa.edu.cn

Received 1 April 2021; Accepted 27 July 2021; Published 18 August 2021

Copyright (c) 2021 Fushou Liu and Dongping Jin. Exclusive Licensee Beijing Institute of Technology Press. Distributed under a Creative Commons Attribution License (CC BY 4.0).

\begin{abstract}
The paper presents a high-efficient finite difference method for solving the PDE model of the single-link flexible manipulator system with boundary feedback control. Firstly, an abstract state-space model of the manipulator is derived from the original PDE model and the associated boundary conditions of the manipulator by using the velocity and bending curvature of the flexible link as the state variables. Then, the second-order implicit Crank-Nicolson scheme is adopted to discretize the statespace equation, and the second-order one-sided approximation is used to discretize the boundary conditions with excitations and feedback control. At last, the state-space equation combined with the boundary conditions of the flexible manipulator is transformed to a system of linear algebraic equations, from which the response of the flexible manipulator can be easily solved. Numerical simulations are carried out to simulate the manipulator under various excitations and boundary feedback control. The results are compared with ANSYS to demonstrate the accuracy and high efficiency of the presented method.
\end{abstract}

\section{Introduction}

Flexible manipulators are widely used in aerospace engineering for the applications of active debris removal, building large space structures, and servicing satellites, etc. [1-3]. In order to meet the requirements of high speed, high accuracy, and low energy consumption, the manipulators tend to be more and more lightweight and highly flexible. As a consequence, the vibration problem has become one of the most important considerations in the design of flexible manipulators [2-4]. As complex distributed parameter systems with coupling dynamics between the rigid and the flexible modes [5], the traditional controller design approach which uses the discretized ordinary differential equation (ODE) models is inaccurate to describe the spatiotemporally varying states and may cause spillover instability due to the neglect of higher-order modes [5, 6]. Alternatively, the controller design for flexible manipulators using the partial differential equation (PDE) models without discretization has gained significant attention in recent years [5-12]. For example, Jiang et al. [5] designed an infinite dimensional disturbance observer for the boundary control of the flexible manipula- tor. Ge et al. [6] introduced nonlinear strain feedback to improve the performance of the boundary PD controller on the flexible manipulator. He et al. [12] designed a boundary controller based on the adaptive inverse algorithm for the flexible manipulator with input backlash.

In order to verify the controller by a numerical simulation or carry out a real-time state estimation in the control experiment, a high-efficient solving method for the PDE model of the flexible manipulator is in demand. The finite difference (FD) method is widely used for solving the PDE model of the control system [5-12]. As a classical numerical method for solving the PDE system, the FD method has been well developed for simulation of flexible structures such as strings $[13,14]$ and beams [15-17]. However, the flexible manipulator incorporating hub inertia and payload is more complex than a single string or beam since it is a coupled PDE-ODE system. Specialized studies on developing the FD method for the single-link flexible manipulator (SLFM) were carried out by Tzes et al. [18] and Tokhi et al. [1921]. They all used the central difference scheme to discretize the original fourth-order PDE model and the associated boundary conditions of the SLFM system. The methods 
presented by Refs. [18-21] have been used by many researchers for flexible manipulators in various applications such as response simulation, trajectory tracing, and parametric identification until now [22-26]. However, the above methods all involve iteration procedures at each time step, and the selection of time step $\Delta t$ and space step $\Delta x$ must satisfy the stability condition, i.e., $\left(\Delta t^{2} E I\right) /\left(\Delta x^{4} \rho A\right) \leq 1 / 4$ ( $E I$ and $\rho A$ are the flexural rigidity and the mass per unit length of the flexible link, respectively), which usually leads to a very small time step and a long computational time. Tokhi and Azad [19] investigated the real-time performance of their algorithm and suggested that the MATLAB package is not suitable for real-time simulation of their algorithm because of the need for large iteration loops. Alternatively, they implemented their algorithm on a parallel computation platform to achieve real-time performance [20]. Another method for solving the highorder PDE is to transfer it to a low-order PDE system by using the state variables; then, a wider variety of difference procedures are available, and high computational efficiency could be obtained. For example, Abhyankar et al. [15] used the bending moment as the state variable to reduce the order of the PDE of a nonlinear EulerBernoulli beam model and obtained a more efficient FD method. However, this approach has not been applied to the solution of the flexible manipulator till now. This paper presents a new FD method for simulation of the SLFM system by transferring the original PDE model to an abstract state-space model and then using the secondorder accurate and unconditionally stable Crank-Nicolson scheme to solve the state-space model. The presented method achieves a very high efficiency since it avoids the needs of large iteration loops and very small time steps as in the previous studies [18-21].

The paper is organized as follows: In Section 2, the PDE model and boundary conditions of SLFM with boundary feedback control are given, and an abstract state-space model is derived from the above PDE model by using the velocity and curvature of the flexible link as state variables. In Section 3 , the Crank-Nicolson scheme and the second-order accurate one-sided approximation are adopted to discretize the statespace equation and the boundary conditions of the SLFM system. Finally, numerical simulations for the SLFM under excitations and boundary feedback control are presented in Section 4.

\section{Abstract State-Space Model of the SLFM}

2.1. Dynamic Model of the SLFM. The single-link flexible manipulator considered in this paper is shown in Figure 1, which consists of a rigid hub, a flexible link with length $L$, and a payload at the free end of the link, in which $X O Y$ is the inertial coordinate and $X_{1} O Y_{1}$ is the rotating coordinate attached to the link. Suppose that the manipulator only moves in the horizontal plane and a control torque $\tau(t)$ and a control force $F(t)$ are applied at the hub and the free end of the manipulator by the joint motor and a force actuator, respectively.

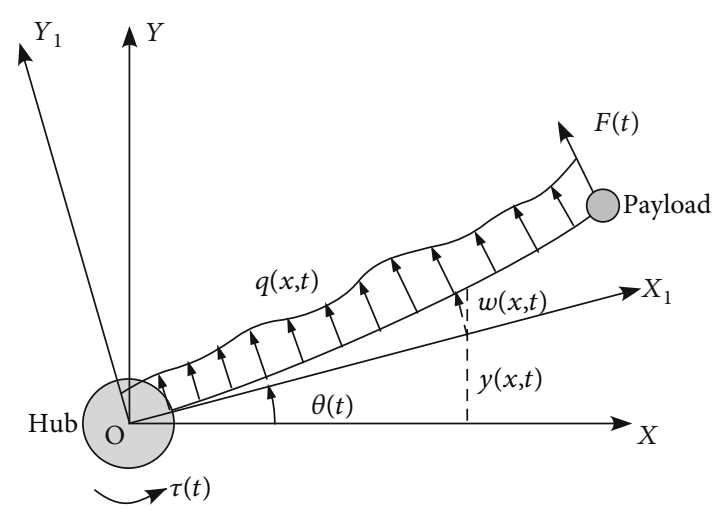

Figure 1: A single-link flexible manipulator.

For an angular displacement $\theta$ of the hub and a small elastic deflection $w$ of the flexible link, the total displacement $y$ at an arbitrary point of the flexible link can be described as

$$
y(x, t)=x \theta(t)+w(x, t) .
$$

Modeling the flexible link as an Euler-Bernoulli beam with structural damping (using the Chen-Russell damping model) [27], the equation of motion for the flexible link can be derived as

$$
\rho A \ddot{y}(x, t)+E I y^{\prime \prime \prime \prime}(x, t)-c_{s} \dot{y}^{\prime \prime}(x, t)=q(x, t),
$$

where $E I$ and $\rho A$ are the flexural rigidity and the mass per unit length of the link, respectively, $c_{s}$ is the coefficient of structural damping, $q(x, t)$ is a distributed spatiotemporally varying disturbance load along the flexible link, and the overdot and prime indicate the derivatives with respect to $t$ and $x$, respectively.

From the natural boundary conditions of the flexible link and the equilibrium equations for the rigid hub and the payload, the boundary conditions of the SLFM system can be derived as [6]

$$
\begin{aligned}
w(0, t) & =w^{\prime}(0, t)=w^{\prime \prime}(L, t)=0, \\
I_{\mathrm{h}} \ddot{\theta}-E I w^{\prime \prime}(0, t) & =\tau(t), \\
m \ddot{y}(L, t)-E I w^{\prime \prime \prime}(L, t) & =F(t),
\end{aligned}
$$

where $I_{\mathrm{h}}$ is the inertia of the hub and $m$ is the mass of the payload. Substituting Equation (1) into Equation (3) yields

$$
\begin{aligned}
y(0, t) & =0, \\
I_{\mathrm{h}} \ddot{y}^{\prime}(0, t)-E I y^{\prime \prime}(0, t) & =\tau(t), \\
y^{\prime \prime}(L, t) & =0, \\
m \ddot{y}(L, t)-E I y^{\prime \prime \prime}(L, t) & =F(t) .
\end{aligned}
$$

2.2. Abstract State-Space Equation of the SLFM. Equation (2) is a fourth-order PDE; it can be discretized directly by using 
the central difference scheme as in Refs. [18-21]; however, this approach yields a recursive equation which needs an iterative algorithm. Note that the velocity and bending curvature of the flexible link can be written as

$$
\left\{\begin{array}{l}
v(x, t)=\dot{y}(x, t) \\
\kappa(x, t)=y^{\prime \prime}(x, t)
\end{array}\right.
$$

Using the variable substitution method, the equation of motion of the manipulator can be written as

$$
\left\{\begin{array}{l}
\dot{v}(x, t)=-a_{1} \kappa^{\prime \prime}(x, t)+a_{2} v^{\prime \prime}(x, t)+\bar{q}(x, t), \\
\dot{\kappa}(x, t)=v^{\prime \prime}(x, t),
\end{array}\right.
$$

where $a_{1}=E I / \rho A, a_{2}=c_{s} / \rho A$, and $\bar{q}(x, t)=q(x, t) / \rho A$.

The boundary conditions in Equation (4) can be expressed with the state variables as

$$
\begin{aligned}
v(0, t) & =0, \\
I_{\mathrm{h}} \dot{v}^{\prime}(0, t)-E I \kappa(0, t) & =\tau(t), \\
\kappa(L, t) & =0, \\
m \dot{v}(L, t)-E I \kappa^{\prime}(L, t) & =F(t) .
\end{aligned}
$$

The above transformation yields lower-order derivatives in the equations of motion as well as in the boundary conditions, which makes the equations much easier to solve by the finite difference method.

Denoting the state vector $\mathbf{x}(x, t)=\{v(x, t), \kappa(x, t)\}^{T}$, then Equation (6) can be recast into the following abstract state equation [26]:

$$
\dot{\mathbf{x}}=\mathbf{A} \mathbf{x}^{\prime \prime}+\mathbf{q}
$$

where

$$
\begin{aligned}
& \mathbf{A}=\left[\begin{array}{cc}
a_{2} & -a_{1} \\
1 & 0
\end{array}\right], \\
& \mathbf{q}=\left\{\begin{array}{c}
\bar{q}(x, t) \\
0
\end{array}\right\} .
\end{aligned}
$$

The abstract state-space model is widely used in the controller and observer design of distributed parameter systems [28]. Since the state variables in Equation (8) are the velocity and strain of the manipulator, this model is much appropriate for the controller design based on velocity feedback or strain feedback such as the studies in Refs. [29-31].

\section{Finite Difference Method for the State-Space Model of SLFM}

3.1. Discretization of the Abstract State Equation. The abstract state Equation (8) is a parabolic PDE system when the damping coefficient $c_{s}>0$ or a Petrovskii parabolic sys- tem when $c_{s}=0$ [32]. For solving this equation with the boundary condition (7a), (7b), (7c), (7d), the implicit and unconditionally stable Crank-Nicolson difference scheme is suitable [32]. The Crank-Nicolson scheme can be used to solve PDEs that are first order in time and arbitrary order in space with mixed partial derivatives; it applies central differencing to the PDEs about the point $(x, t+\Delta t / 2)$ and evaluates the time derivate and the space derivate of an arbitrary function $u(x, t)$ as [32]

$$
\begin{aligned}
\dot{u}\left(x, t+\frac{1}{2} \Delta t\right)= & \frac{u(x, t+\Delta t)-u(x, t)}{\Delta t}+O\left(\Delta t^{2}\right) \\
u^{\prime}\left(x, t+\frac{1}{2} \Delta t\right)= & \frac{1}{2}\left[\frac{u(x+\Delta x, t+\Delta t)-u(x-\Delta x, t+\Delta t)}{2 \Delta x}\right. \\
& +u(x+\Delta x, t)-u\left(\frac{x-\Delta x, t)}{2 \Delta x}\right]+O\left(\Delta t^{2}\right)+O\left(\Delta x^{2}\right) .
\end{aligned}
$$

It has second-order accuracy in both time and space. This stability property can be utilized to take large time steps in the simulation.

For the abstract state Equation (8), discretizing the spatial variable as $x_{m}=m \Delta x(m=0,1,2, \cdots, N+1)$, and the time as $t_{n}=n \Delta t \quad(n=0,1,2, \cdots)$, then by utilizing the CrankNicolson scheme, it can be discretized as

$$
\begin{aligned}
\frac{\mathbf{x}_{m}^{n+1}-\mathbf{x}_{m}^{n}}{\Delta t}= & \frac{1}{2} \mathbf{A} \frac{\mathbf{x}_{m+1}^{n+1}-2 \mathbf{x}_{m}^{n+1}+\mathbf{x}_{m-1}^{n+1}}{\Delta x^{2}} \\
& +\frac{1}{2} \mathbf{A} \frac{\mathbf{x}_{m+1}^{n}-2 \mathbf{x}_{m}^{n}+\mathbf{x}_{m-1}^{n}}{\Delta x^{2}} \\
& +\frac{\mathbf{q}_{m}^{n+1}+\mathbf{q}_{m}^{n}}{2}(m=1,2, \cdots, N, n=0,1,2, \cdots),
\end{aligned}
$$

where $\mathbf{x}_{m}^{n} \equiv \mathbf{x}\left(x_{m}, t_{n}\right), \mathbf{q}_{m}^{n} \equiv \mathbf{q}\left(x_{m}, t_{n}\right)$.

Letting $\mu=\Delta t / \Delta x^{2}$, Equation (11) can be rewritten as

$$
\begin{aligned}
& -\mu \mathbf{A} \mathbf{x}_{m-1}^{n+1}+2(\mathbf{I}+\mu \mathbf{A}) \mathbf{x}_{m}^{n+1}-\mu \mathbf{A} \mathbf{x}_{m+1}^{n+1} \\
& \quad=\mu \mathbf{A} \mathbf{x}_{m-1}^{n}+2(\mathbf{I}-\mu \mathbf{A}) \mathbf{x}_{m}^{n}+\mu \mathbf{A} \mathbf{x}_{m+1}^{n}+\Delta t\left(\mathbf{q}_{m}^{n+1}+\mathbf{q}_{m}^{n}\right),
\end{aligned}
$$

where $\mathbf{I}$ is a $2 \times 2$ identity matrix. At the time step $n+1$, the state vector at the previous time step and the load vector at the current time step are assumed known, so Equation (12) can be simplified as

$$
-\mu \mathbf{A} \mathbf{x}_{m-1}^{n+1}+2(\mathbf{I}+\mu \mathbf{A}) \mathbf{x}_{m}^{n+1}-\mu \mathbf{A} \mathbf{x}_{m+1}^{n+1}=\mathbf{b}_{m}^{n+1},
$$

where

$$
\mathbf{b}_{m}^{n+1}=\mu \mathbf{A} \mathbf{x}_{m-1}^{n}+2(\mathbf{I}-\mu \mathbf{A}) \mathbf{x}_{m}^{n}+\mu \mathbf{A} \mathbf{x}_{m+1}^{n}+\Delta t\left(\mathbf{q}_{m}^{n+1}+\mathbf{q}_{m}^{n}\right)
$$

is known at the current time step. 
By discretizing the state equation at each interior point $x_{m}(m=1,2, \cdots, N)$, the following system of equations can be obtained:

$$
\left\{\begin{array}{l}
-\mu \mathbf{A} \mathbf{x}_{0}^{n+1}+2(\boldsymbol{I}+\mu \mathbf{A}) \mathbf{x}_{1}^{n+1}-\mu \mathbf{A} \mathbf{x}_{2}^{n+1}=\mathbf{b}_{1}^{n+1} \\
-\mu \mathbf{A} \mathbf{x}_{1}^{n+1}+2(\boldsymbol{I}+\mu \mathbf{A}) \mathbf{x}_{2}^{n+1}-\mu \mathbf{A} \mathbf{x}_{3}^{n+1}=\mathbf{b}_{2}^{n+1} \\
\vdots \\
-\mu \mathbf{A} \mathbf{x}_{N-2}^{n+1}+2(\boldsymbol{I}+\mu \mathbf{A}) \mathbf{x}_{N-1}^{n+1}-\mu \mathbf{A} \mathbf{x}_{N}^{n+1}=\mathbf{b}_{N-1}^{n+1} \\
-\mu \mathbf{A} \mathbf{x}_{N-1}^{n+1}+2(\boldsymbol{I}+\mu \mathbf{A}) \mathbf{x}_{N}^{n+1}-\mu \mathbf{A} \mathbf{x}_{N+1}^{n+1}=\mathbf{b}_{N}^{n+1}
\end{array}\right.
$$

Equation (15) is a system of linear algebraic equations containing $2 N$ equations and $2 N+4$ unknown variables, which cannot be solved directly. However, there are four unknown variables that can be determined directly or calculated in terms of their neighboring values from the four boundary conditions in Equation (7a), (7b), (7c), (7d).

3.2. Discretization of the Boundary Conditions. The boundary conditions (7a) and (7c) give

$$
\begin{gathered}
v_{0}^{n+1}=0, \\
\kappa_{N+1}^{n+1}=0 .
\end{gathered}
$$

According to the Crank-Nicolson scheme, the boundary condition $(7 \mathrm{~b})$ is differenced about the point $\left(0, t_{n}+\Delta t / 2\right)$, which yields

$$
I_{\mathrm{h}} \dot{v}^{\prime}\left(0, t_{n}+\frac{\Delta t}{2}\right)-E I \kappa\left(0, t_{n}+\frac{\Delta t}{2}\right)=\tau\left(t_{n}+\frac{\Delta t}{2}\right) .
$$

It follows from Equation (18) that

$\frac{I_{h}}{\Delta t}\left[v^{\prime}\left(0, t_{n+1}\right)-v^{\prime}\left(0, t_{n}\right)\right]-\frac{E I}{2}\left(\kappa_{0}^{n+1}+\kappa_{0}^{n}\right)=\frac{1}{2}\left(\tau_{0}^{n+1}+\tau_{0}^{n}\right)$.

Furthermore, calculating the spatial derivative at the boundary $x=0$ by using the second-order accurate onesided approximation method yields [32]

$$
v^{\prime}\left(0, t_{n}\right)=\frac{-3 v_{0}^{n}+4 v_{1}^{n}-v_{2}^{n}}{2 \Delta x}
$$

Substituting Equation (20) into (19) gives

$\kappa_{0}^{n+1}=-\kappa_{0}^{n}+b_{1}\left(4 v_{1}^{n+1}-v_{2}^{n+1}\right)-b_{1}\left(4 v_{1}^{n}-v_{2}^{n}\right)-\frac{1}{E I}\left(\tau^{n+1}+\tau^{n}\right)$,

where $b_{1}=I_{\mathrm{h}} / E I \Delta t \Delta x$.
Taking a similar difference procedure for the boundary condition $(7 \mathrm{~d})$ yields

$\frac{m}{\Delta t}\left(v_{N+1}^{n+1}-v_{N+1}^{n}\right)-\frac{E I}{2}\left[\kappa^{\prime}\left(L, t_{n+1}\right)+\kappa^{\prime}\left(L, t_{n}\right)\right]=\frac{1}{2}\left(F^{n+1}+F^{n}\right)$.

Noticing that the spatial derivative at the right boundary is approximated by [30],

$$
\kappa^{\prime}\left(L, t_{n}\right)=\frac{-\left(-3 \kappa_{N+1}^{n}+4 \kappa_{N}^{n}-\kappa_{N-1}^{n}\right)}{2 \Delta x} .
$$

At last, the difference formula for the boundary condition (7d) can be obtained as

$$
\begin{aligned}
v_{N+1}^{n+1}= & v_{N+1}^{n}-\frac{b_{2}}{4}\left[\left(4 \kappa_{N}^{n+1}-\kappa_{N-1}^{n+1}\right)+\left(4 \kappa_{N}^{n}-\kappa_{N-1}^{n}\right)\right] \\
& +\frac{\Delta t}{2 m}\left(F^{n+1}+F^{n}\right)
\end{aligned}
$$

where $b_{2}=E I \Delta t / m \Delta x$.

3.3. Solution of the System of Equations. For making use of the boundary condition at the left end of the flexible link, the first equation in Equation (15) is rewritten as

$$
-\mu \mathbf{A t}_{1} v_{0}^{n+1}-\mu \mathbf{A} \mathbf{t}_{2} \kappa_{0}^{n+1}+2(\mathbf{I}+\mu \mathbf{A}) \mathbf{x}_{1}^{n+1}-\mu \mathbf{A} \mathbf{x}_{2}^{n+1}=\mathbf{b}_{1}^{n},
$$

where $\mathbf{t}_{1}=\{1,0\}^{T}, \mathbf{t}_{2}=\{0,1\}^{T}$.

Substituting the boundary conditions (16) and (20) into Equation (25) yields

$$
\mathbf{A}_{d}^{11} \mathbf{x}_{1}^{n+1}+\mathbf{A}_{d}^{12} \mathbf{x}_{2}^{n+1}=\widehat{\mathbf{b}}_{1}^{n+1}
$$

where

$$
\begin{aligned}
\mathbf{A}_{d}^{11} & =2(\mathbf{I}+\mu \mathbf{A})-4 b_{1} \mu \mathbf{A} \mathbf{t}_{2} \mathbf{t}_{1}^{T}, \\
\mathbf{A}_{d}^{12} & =-\left(\mu \mathbf{A}-b_{1} \mu \mathbf{A} \mathbf{t}_{2} \mathbf{t}_{1}^{\mathrm{T}}\right), \\
\widehat{\mathbf{b}}_{1}^{n+1} & =\mathbf{b}_{1}^{n+1}-\mu \mathbf{A t}_{2}\left[\kappa_{0}^{n}+b_{1}\left(4 v_{1}^{n}-v_{2}^{n}\right)+\frac{1}{E I}\left(\tau^{n+1}+\tau^{n}\right)\right] .
\end{aligned}
$$

For making use of the boundary condition at the right end of the flexible link, the last equation in Equation (15) is rewritten as

$$
-\mu \mathbf{A} \mathbf{x}_{N-1}^{n+1}+2(\mathbf{I}+\mu \mathbf{A}) \mathbf{x}_{N}^{n+1}-\mu \mathbf{A t}_{1} v_{N+1}^{n+1}-\mu \mathbf{A t}_{2} \kappa_{N+1}^{n+1}=\mathbf{b}_{N}^{n+1} .
$$

Substituting the boundary conditions (17) and (24) into Equation (28) gives

$$
\mathbf{A}_{d}^{N(N-1)} \mathbf{x}_{N-1}^{n+1}+\mathbf{A}_{d}^{N N} \mathbf{x}_{N}^{n+1}=\widehat{\mathbf{b}}_{N}^{n+1},
$$




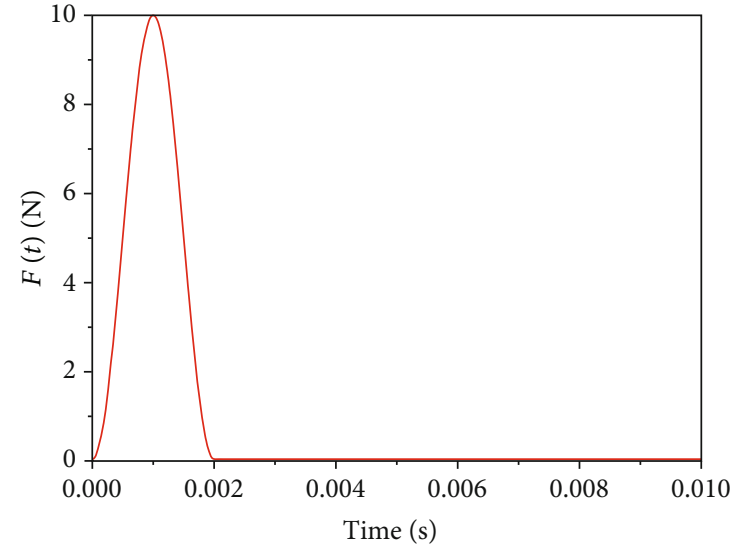

FIgURe 2: A broadband impulse load with frequency bandwidth $1 \mathrm{kHz}$.

where

$$
\begin{aligned}
\mathbf{A}_{d}^{N(N-1)} & =-\left(\mu \mathbf{A}+\frac{1}{4} \mu b_{2} \mathbf{A t}_{1} \mathbf{t}_{2}^{\mathrm{T}}\right), \\
\mathbf{A}_{d}^{N N} & =2(\mathbf{I}+\mu \mathbf{A})+\mu b_{2} \mathbf{A t}_{1} \mathbf{t}_{2}^{T}, \\
\widehat{\mathbf{b}}_{N}^{n+1} & =\mathbf{b}_{N}^{n+1}+\mu \mathbf{A t}_{1}\left[v_{N+1}^{n}-\frac{b_{2}}{4}\left(4 \kappa_{N}^{n}-\kappa_{N-1}^{n}\right)+\frac{\Delta t}{2 m}\left(F^{n+1}+F^{n}\right)\right] .
\end{aligned}
$$

With the above transformation, the systems of equations in Equation (15) can be replaced by new systems of equations which contains $2 \mathrm{~N}$ equations and $2 \mathrm{~N}$ unknown variables as

$$
\mathbf{A}_{d} \mathbf{x}^{n+1}=\mathbf{b}^{n+1}
$$

where

$$
\mathbf{A}_{d}=\left[\begin{array}{ccccccc}
\mathbf{A}_{d}^{11} & \mathbf{A}_{d}^{12} & \mathbf{0} & \mathbf{0} & \mathbf{0} & \cdots & \mathbf{0} \\
-\mu \mathbf{A} & 2(\mathbf{I}+\mu \mathbf{A}) & -\mu \mathbf{A} & \mathbf{0} & \mathbf{0} & \ldots & \mathbf{0} \\
\mathbf{0} & -\mu \mathbf{A} & 2(\mathbf{I}+\mu \mathbf{A}) & -\mu \mathbf{A} & \mathbf{0} & \ldots & \mathbf{0} \\
\vdots & \ddots & \ddots & \ddots & \ddots & \ddots & \vdots \\
\mathbf{0} & \cdots & \mathbf{0} & -\mu \mathbf{A} & 2(\mathbf{I}+\mu \mathbf{A}) & -\mu \mathbf{A} & \mathbf{0} \\
\mathbf{0} & \mathbf{0} & \cdots & \mathbf{0} & -\mu \mathbf{A} & 2(\mathbf{I}+\mu \mathbf{A}) & -\mu \mathbf{A} \\
\mathbf{0} & \mathbf{0} & \mathbf{0} & \cdots & \mathbf{0} & \mathbf{A}_{d}^{N(N-1)} & \mathbf{A}_{d}^{N N}
\end{array}\right],
$$

$$
\mathbf{x}^{n+1}=\left\{\begin{array}{c}
\mathbf{x}_{1}^{n+1} \\
\mathbf{x}_{2}^{n+1} \\
\mathbf{x}_{3}^{n+1} \\
\vdots \\
\mathbf{x}_{N-2}^{n+1} \\
\mathbf{x}_{N-1}^{n+1} \\
\mathbf{x}_{N}^{n+1}
\end{array}\right\},
$$
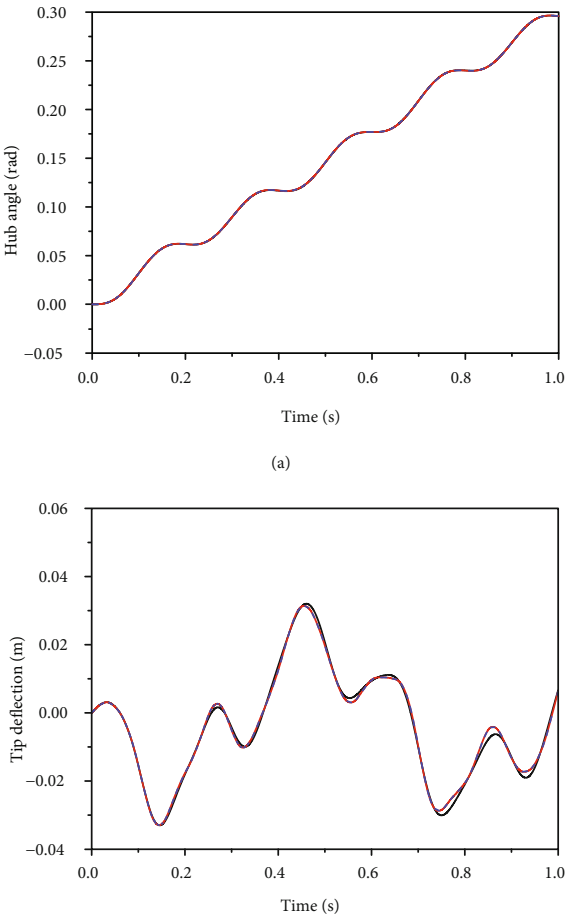

(b)

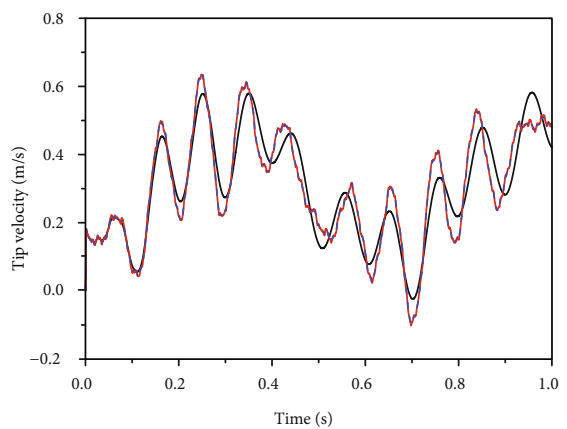

(c)

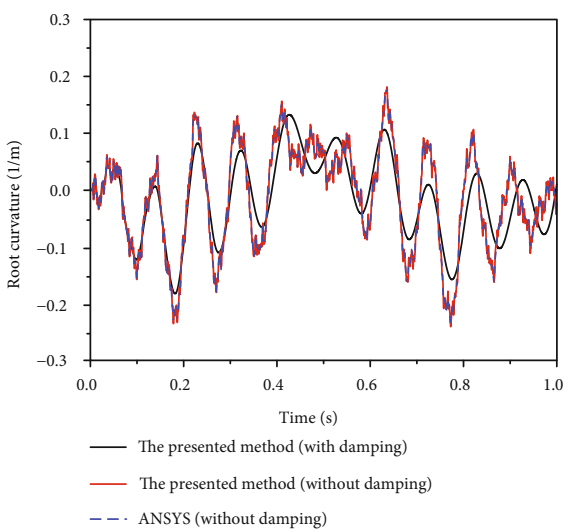

FIGURE 3: Forced vibration response of the manipulator under harmonic loads and a broadband impulse load: (a) the hub angle; (b) the tip deflection; (c) the tip velocity; (d) the root curvature. 
TABLE 1: Comparison of the computational efficiency between the presented method and ANSYS.

\begin{tabular}{lccr}
\hline & Simulation parameters & The presented method & ANSYS \\
\hline With impulse load & $\Delta t=1 \times 10^{-4} \mathrm{~s}, \Delta x=L / 100$ & About $8 \mathrm{~s}$ & About $22 \mathrm{~min}$ \\
& $\Delta t=1 \times 10^{-4} \mathrm{~s}, \Delta x=L / 20$ & About $1.4 \mathrm{~s}$ & About $16 \mathrm{~min}$ \\
Without impulse load & $\Delta t=1 \times 10^{-3} \mathrm{~s}, \Delta x=L / 100$ & About $0.7 \mathrm{~s}$ & About $3.5 \mathrm{~min}$ \\
& $\Delta t=1 \times 10^{-3} \mathrm{~s}, \Delta x=L / 20$ & About $0.3 \mathrm{~s}$ & About $2.5 \mathrm{~min}$ \\
\hline
\end{tabular}

$\mathbf{b}^{n+1}=\left\{\begin{array}{c}\widehat{\mathbf{b}}_{1}^{n+1} \\ \mathbf{b}_{2}^{n+1} \\ \mathbf{b}_{3}^{n+1} \\ \vdots \\ \mathbf{b}_{N-2}^{n+1} \\ \mathbf{b}_{N-1}^{n+1} \\ \widehat{\mathbf{b}}_{N}^{n+1}\end{array}\right\}$.

Equation (32) is a tridiagonal system of equations, which can be solved directly by evaluating the inverse of matrix $\mathbf{A}_{d}$ or be solved more efficiently by using the Thomas algorithm [32].

From the velocity of an arbitrary point at the flexible link, the displacement $y$ with second-order accuracy can be obtained by using the average velocity as [15]

$$
y_{m}^{n+1}=y_{m}^{n}+\frac{\Delta t}{2}\left(v_{m}^{n}+v_{m}^{n+1}\right)
$$

Furthermore, the hub angle can be obtained by using Equation (1) and the second-order accurate one-sided approximation as

$$
\theta^{n+1}=y^{\prime}\left(0, t_{n+1}\right) \approx \frac{-3 y_{0}^{n+1}+4 y_{1}^{n+1}-y_{2}^{n+1}}{2 \Delta x}
$$

3.4. Solution of the SLFM with Boundary Feedback Control. Considering the position and vibration control of the SLFM using linear boundary feedback control, the proportional derivative and strain (PDS) control [29] is selected. The deriving torque is obtained as

$$
\begin{aligned}
\tau(t) & =-k_{p}\left[\theta(t)-\theta_{d}\right]-k_{v} \dot{\theta}(t)-k_{e} w^{\prime \prime}(0, t) \\
& =-k_{p}\left[y^{\prime}(0, t)-\theta_{d}\right]-k_{v} v^{\prime}(0, t)-k_{e} \kappa(0, t),
\end{aligned}
$$

where $\theta_{d}$ is the designed angular position of the manipulator, $k_{p}$ and $k_{v}$ are the feedback gains of the PD controller, and $k_{e}$ is the strain feedback gain.

Substituting Equation (37) into the boundary condition (7b) yields

$I_{h} \dot{v}^{\prime}(0, t)+k_{v} v^{\prime}(0, t)-\left(E I-k_{e}\right) \kappa(0, t)+k_{p}\left[y^{\prime}(0, t)-\theta_{d}\right]=0$. yields

Differencing Equation (38) about the point $\left(0, t_{n}+\Delta t / 2\right)$

$$
\begin{aligned}
& I_{h} \dot{v}^{\prime}\left(0, t_{n}+\frac{\Delta t}{2}\right)+k_{v} v^{\prime}\left(0, t_{n}+\frac{\Delta t}{2}\right)-\left(E I-k_{e}\right) \kappa\left(0, t_{n}+\frac{\Delta t}{2}\right) \\
& \quad+k_{p}\left[y^{\prime}\left(0, t_{n}+\frac{\Delta t}{2}\right)-\theta_{d}\right]=0 .
\end{aligned}
$$

From Equation (39) and utilizing Equation (35), it can be obtained that

$$
\begin{aligned}
& \frac{I_{h}}{\Delta t}\left[v^{\prime}\left(0, t_{n+1}\right)-v^{\prime}\left(0, t_{n}\right)\right]+\frac{k_{v}}{2}\left[v^{\prime}\left(0, t_{n+1}\right)+v^{\prime}\left(0, t_{n}\right)\right] \\
& \quad-\frac{E I-k_{e}}{2}\left(\kappa_{0}^{n+1}+\kappa_{0}^{n}\right) \\
& \quad+k_{p}\left[y^{\prime}\left(0, t_{n}\right)+\frac{\Delta t}{4} v^{\prime}\left(0, t_{n}\right)+\frac{\Delta t}{4} v^{\prime}\left(0, t_{n+1}\right)-\theta_{d}\right]=0 .
\end{aligned}
$$

Calculating the spatial derivative at the boundary $x=0$ in Equation (40) by the second-order accurate one-sided approximation method as in Equation (20) yields

$$
\begin{aligned}
\kappa_{0}^{n+1}= & -\kappa_{0}^{n}+b_{3}\left(4 v_{1}^{n+1}-v_{2}^{n+1}\right)-b_{4}\left(4 v_{1}^{n}-v_{2}^{n}\right) \\
& +b_{5}\left[\left(4 y_{1}^{n}-y_{2}^{n}\right)-2 \theta_{d} \Delta x\right],
\end{aligned}
$$

where

$$
\begin{aligned}
& b_{3}=\frac{1}{\left(E I-k_{e}\right) \Delta x}\left(\frac{I_{h}}{\Delta t}+\frac{k_{v}}{2}+\frac{k_{p} \Delta t}{4}\right), \\
& b_{4}=\frac{1}{\left(E I-k_{e}\right) \Delta x}\left(\frac{I_{h}}{\Delta t}-\frac{k_{v}}{2}-\frac{k_{p} \Delta t}{4}\right), \\
& b_{5}=\frac{k_{p}}{\left(E I-k_{e}\right) \Delta x} .
\end{aligned}
$$

Substituting Equation (41) into Equation (25) yields

$$
\mathbf{A}_{d}^{11} \mathbf{x}_{1}^{n+1}+\mathbf{A}_{d}^{12} \mathbf{x}_{2}^{n+1}=\widehat{\mathbf{b}}_{1}^{n+1}
$$

where

$$
\begin{aligned}
\mathbf{A}_{d}^{11} & =2(\mathbf{I}+\mu \mathbf{A})-4 b_{3} \mu \mathbf{A} \mathbf{t}_{2} \mathbf{t}_{1}^{\mathrm{T}}, \\
\mathbf{A}_{d}^{12} & =-\left(\mu \mathbf{A}-b_{3} \mu \mathbf{A} \mathbf{t}_{2} \mathbf{t}_{1}^{T}\right) \\
\widehat{\mathbf{b}}_{1}^{n+1} & =\mathbf{b}_{1}^{n+1}-\mu \mathbf{A t}_{2}\left\{\kappa_{0}^{n}+b_{4}\left(4 v_{1}^{n}-v_{2}^{n}\right)+b_{5}\left[2 \theta_{d} \Delta x-\left(4 y_{1}^{n}-y_{2}^{n}\right)\right]\right\}
\end{aligned}
$$




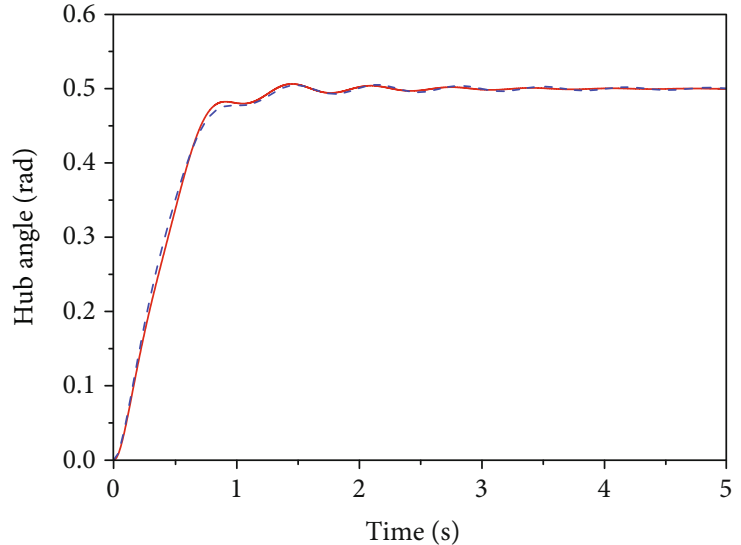

(a)

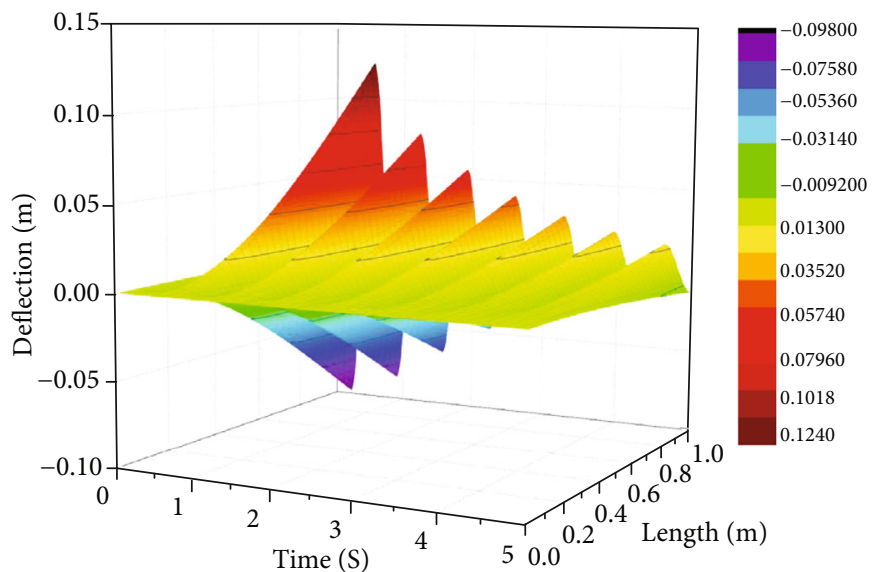

(c)

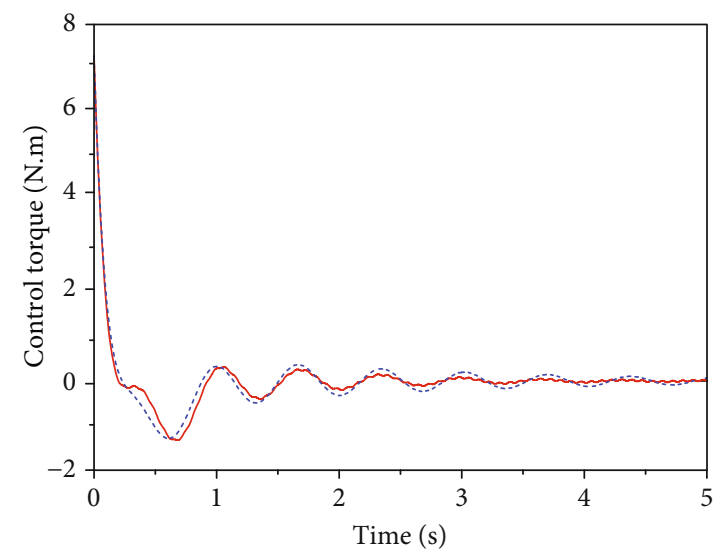

(e)

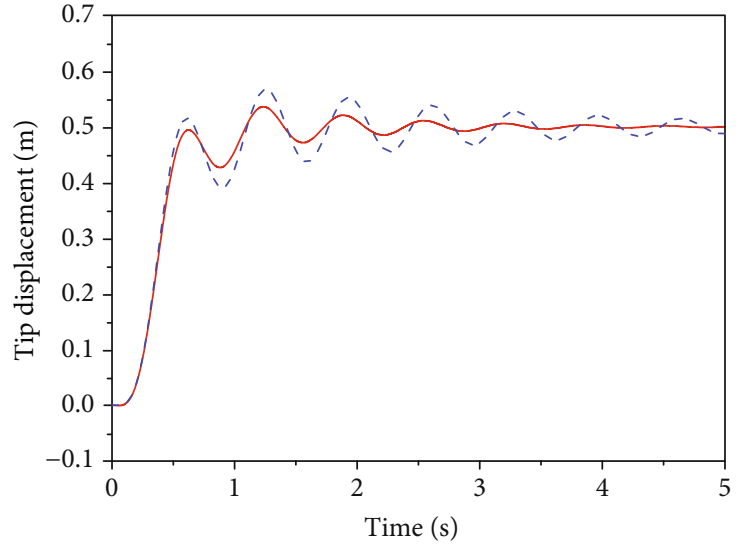

(b)

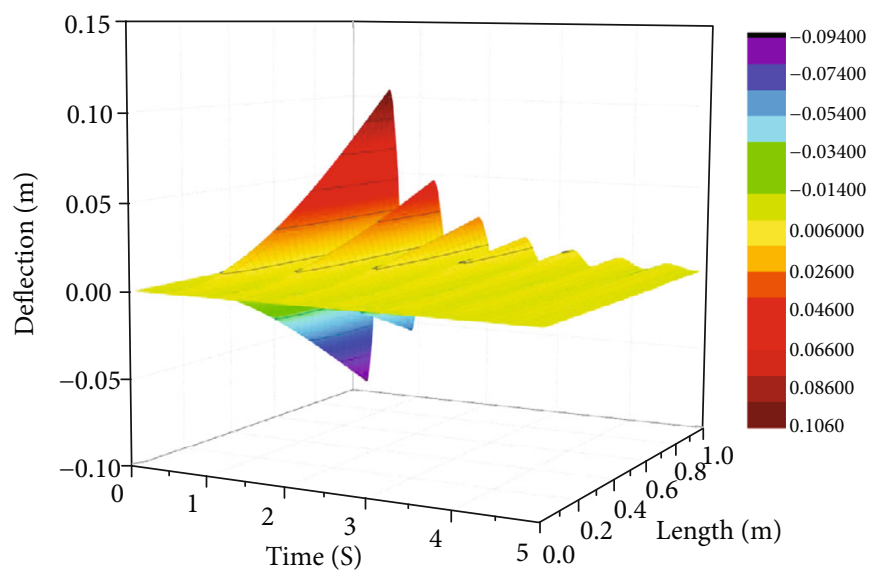

(d)

--- PD control
- PDS control

Figure 4: Simulation results of the manipulator with feedback control: (a) the hub angle; (b) the tip displacement; (c) the deflection of the flexible link with PD control; (d) the deflection of the flexible link with PDS control; (e) the control torque.

Modifying $\mathbf{A}_{d}^{11}, \mathbf{A}_{d}^{12}$, and $\widehat{\mathbf{b}}_{1}^{n+1}$ in Equations (32) and (34) by using Equations (44), (45), and (46), the system of equations for the SLFM with boundary feedback control is obtained.

\section{Results and Discussions}

To verify the correctness and efficiency of the presented method, a numerical study is performed. The system 


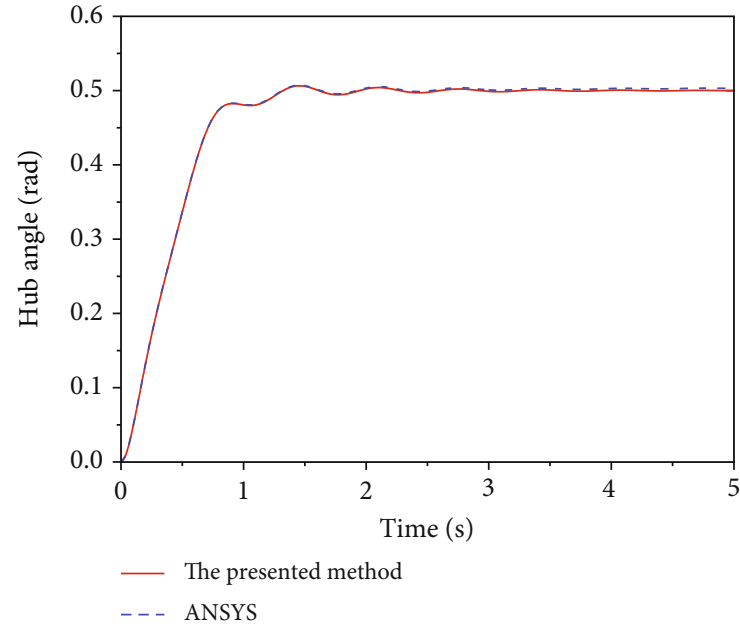

(a)

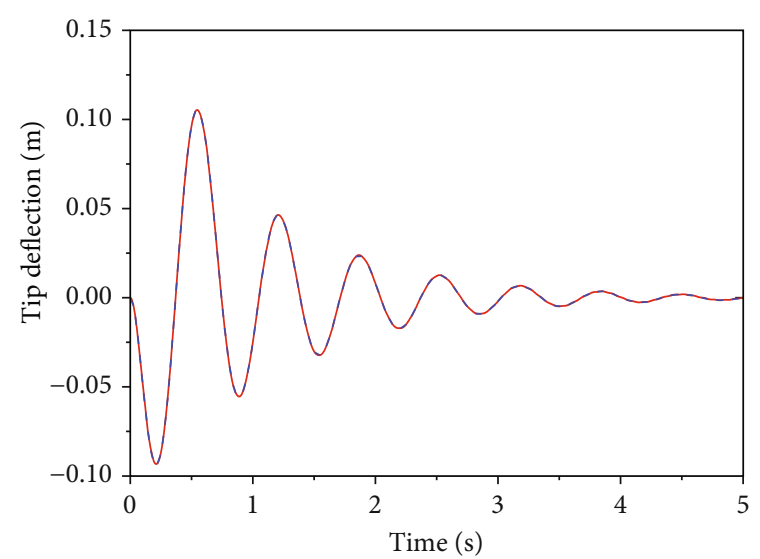

(b)

FIGURE 5: Verification of the control torque of the PDS controller by the ANSYS model: (a) the hub angle; (b) the tip deflection.

parameters of the flexible manipulator are as follows [6]: the flexural rigidity $E I=2.0 \mathrm{~N} \cdot \mathrm{m}^{2}$, the mass per unit length $\rho A=0.1 \mathrm{~kg} / \mathrm{m}$, the length of the link $L=1 \mathrm{~m}$, the inertia of the hub $I_{\mathrm{h}}=0.5 \mathrm{~kg} \cdot \mathrm{m}^{2}$, and the mass of the payload $m=$ $0.05 \mathrm{~kg}$. The damping coefficient $c_{s}$ is assumed to be $0.05 \mathrm{~N}$.s. Since the presented method is based on the unconditional stable Crank-Nicolson scheme, it has no stability requirement on the time step and the space step. The selection of these two simulation parameters is determined by the excitation frequency and the requirement of accuracy.

The presented method is carried out in the MATLAB platform. Its accuracy and performance are verified by comparing with the finite element method. The finite element model of the flexible manipulator is established in ANSYS, in which the flexible link is discretized by planar beam (Beam3) elements and the rigid hub and the payload are modelled as MASS21 elements. The mesh size of the flexible link in the ANSYS model is identical to the space step used in the finite difference method, and the dynamic analysis in ANSYS uses the same time step as in the finite difference method. Since the Chen-Russell damping model is difficult to model in ANSYS, during comparison of the presented method and ANSYS, the damping is neglected.

To demonstrate the correctness of the presented method for SLFM under various loading conditions, both of the low-frequency excitation and the high-frequency excitation are applied simultaneously on the manipulator. For the low-frequency excitation, a driving torque $\tau(t)=5 \sin (2 \pi$. $5 t) \mathrm{N} \cdot \mathrm{m}$ and a distributed disturbance load $q(x, t)=0.5 \sin$ $(2 \pi \cdot 10 t) \mathrm{N} / \mathrm{m}$ are applied. For the high-frequency excitation, a broadband impulse load $F(t)$ is applied on the payload with [33]

$$
F(t)=F_{0}\left[H(t)-H\left(t-\frac{2}{f}\right)\right] \frac{1-\cos (\pi f t)}{2},
$$

where $H(t)$ is the Heaviside function and $f$ is the bandwidth. In this simulation, $F_{0}=10 \mathrm{~N}$ and $f=1 \mathrm{kHz}$ are selected; the corresponding impulse load is shown in Figure 2. The time step and space step for this simulation are set as $\Delta t=1 \times$ $10^{-4} \mathrm{~s}$ and $\Delta x=L / 100=0.01 \mathrm{~m}$, respectively.

The responses of the flexible manipulator under the above excitations are shown in Figure 3. Comparing the results of the presented method and ANSYS for the undamped flexible manipulator, it can be found that they agree very well, which verifies the correctness of the presented method for simulating the SLFM under both lowand high-frequency excitations. Furthermore, it can be found that the damping of the flexible link has a negligible influence on the hub angel but has an obvious effect on the vibration of the flexible link.

The comparison of computational efficiencies of the presented method and ANSYS using different simulation parameters is listed in Table 1. It can be found that the computational times of the presented method are much less than those of ANSYS in all simulation cases, which shows the extremely high efficiency of the presented method. Moreover, it can be found that if a large time step is used when there is no high-frequency impulse load applied on the flexible manipulator, the computational time of the presented method is smaller than the simulation time, which indicates that the presented method can satisfy the requirement of real-time simulation of the flexible manipulator if no highfrequency loads are applied.

Next, the position and vibration control of the SLFM using the boundary controller in Equation (39) are simulated. The desired angular position of manipulator is $\theta_{d}=0.5 \mathrm{rad}$. The gains of the PD controller are set as $k_{p}=14.58 \mathrm{~N} \cdot \mathrm{m} / \mathrm{rad}$ and $k_{v}=5.83 \mathrm{~N} \cdot \mathrm{m} \cdot \mathrm{s} / \mathrm{rad}[6]$; the strain feedback gain is selected as $k_{e}=-E I=-2 \mathrm{~N} \cdot \mathrm{m}^{2}$. The time step for this simulation is selected as $\Delta t=1 \times 10^{-3} \mathrm{~s}$. In order to verify the results of the presented method by ANSYS, the damping of the flexible link is neglected here. The simulation results of the hub angle, the tip displacement, the deflection of the flexible link, 
and the control torque under the PD and PDS controllers are shown in Figure 4. It can be found that the manipulator is driven to the desired position and the vibration of the manipulator is suppressed effectively by both of the two controllers, and the PDS controller can obtain better performance than the PD controller due to the incorporation of strain feedback.

To verify the results of the SLFM with boundary control evaluated by the presented method, the driving torque $\tau(t)$ of the PDS control obtained by the presented method is applied to the ANSYS model, and the response of the manipulator under control was evaluated. The hub angle and tip deflection evaluated by ANSYS are shown in Figure 5. It can be found that the results of the ANSYS model agree well with those of the presented method, which demonstrates the correctness of the presented method for simulating the SLFM with boundary control.

\section{Conclusions}

In this research, a high-efficient finite difference method for solution of the PDE model of single-link flexible manipulators with boundary feedback control was proposed based on the unconditional stable Crank-Nicolson scheme. Numerical simulations verify the correctness and extremely high computational efficiency of the present method for simulating the single-link flexible manipulators under both low- and high-frequency excitations and the PDS (proportional derivative and strain) boundary feedback control. The presented method can satisfy the requirement of real-time simulation for the single-link flexible manipulators if no high-frequency excitations are applied. In the future, the extension of the presented method to the solution of more sophisticated control systems such as the nonlinear rigidflexible coupled models with advanced controllers can be studied $[34,35]$. Meanwhile, the application of the presented method in real-time state estimation of the flexible manipulator can be investigated.

\section{Data Availability}

The data used to support the findings of this study are available from the corresponding author upon request.

\section{Conflicts of Interest}

The authors declared that they have no conflicts of interest to this work.

\section{Acknowledgments}

This work was supported by the National Natural Science Foundation of China under Grants 11702146, 11732006, and 11827801 .

\section{References}

[1] A. Ellery, "Tutorial review on space manipulators for space debris mitigation," Robotics, vol. 8, no. 34, pp. 1-56, 2019.

[2] M. Sabatini, P. Gasbarri, R. Monti, and G. B. Palmerini, "Vibration control of a flexible space manipulator during on orbit operations," Acta Astronautica, vol. 73, no. 2, pp. 109121, 2012.

[3] J. Warminski, L. Kloda, and S. Lenci, "Nonlinear vibrations of an extensional beam with tip mass in slewing motion," Meccanica, vol. 55, no. 12, pp. 2311-2335, 2020.

[4] B. Chen, J. Huang, and J. C. Ji, "Control of flexible single-link manipulators having Duffing oscillator dynamics," Mechanical Systems and Signal Processing, vol. 121, no. 15, pp. 44-57, 2019.

[5] T. Jiang, J. Liu, and W. He, "Boundary control for a flexible manipulator based on infinite dimensional disturbance observer," Journal of Sound and Vibration, vol. 348, pp. 114, 2015.

[6] S. S. Ge, T. H. Lee, and G. Zhu, "Improving regulation of a single-link flexible manipulator with strain feedback," IEEE Transaction on Robotics and Automation, vol. 14, no. 1, pp. 179-185, 1998.

[7] Z. J. Liu, J. K. Liu, and W. He, "Adaptive boundary control of a flexible manipulator with input saturation," International Journal of Control, vol. 89, no. 6, pp. 1191-1202, 2016.

[8] J. T. Ma, Z. T. Wei, H. Wen, and D. Jin, "Boundary control of a Timoshenko beam with prescribed performance," Acta Mechanica, vol. 231, no. 8, pp. 3219-3234, 2020.

[9] X. He, S. Zhang, Y. Ouyang, and Q. Fu, "Vibration control for a flexible single-link manipulator and its application," IET Control Theory and Applications, vol. 14, no. 7, pp. 930-938, 2020.

[10] V. E. M. Kabganian, "Adaptive trajectory control and dynamic friction compensation for a flexible-link robot," Journal of Mechanics, vol. 26, no. 2, pp. 205-217, 2010.

[11] Y. H. Song, W. He, X. Y. He, and Z. Han, "Vibration control of a high-rise building structure: theory and experiment," IEEE/CAA Journal of Automatica Sinica, vol. 8, no. 4, pp. 866-875, 2021.

[12] W. He, X. He, M. Zou, and H. Li, "PDE model-based boundary control design for a flexible robotic manipulator with input backlash," IEEE Transactions on Control Systems Technology, vol. 27, no. 2, pp. 790-797, 2019.

[13] W. J. Zhao, L. Q. Chen, and W. Z. Jean, “A finite difference method for simulating transverse vibrations of an axially moving viscoelastic string," Applied Mathematics and Mechanics (English Edition), vol. 27, no. 1, pp. 23-28, 2006.

[14] S. Zhang, W. He, and D. Huang, "Active vibration control for a flexible string system with input backlash," IET Control Theory and Application, vol. 10, no. 7, pp. 800-805, 2016.

[15] N. S. Abhyankar, E. K. Hall, and S. V. Hanagud, "Chaotic vibrations of beams: numerical solution of partial differential equations," Journal of Applied Mechanics, vol. 60, no. 1, pp. 167-174, 1993.

[16] F. Li and Z. Sun, "A finite difference scheme for solving the Timoshenko beam equations with boundary feedback," Journal of Computational and Applied Mathematics, vol. 200, no. 2, pp. 606-627, 2007.

[17] A. Schirrer, E. Talic, G. Aschauer, M. Kozek, and S. Jakubek, "Optimization based determination of highly absorbing boundary conditions for linear finite difference schemes," Journal of Sound and Vibration, vol. 365, pp. 45-69, 2016.

[18] A. P. Tzes, S. Yurkovich, and F. D. Langer, "A method for solution of the Euler-Bernoulli beam equation in flexible-link robotic systems," in Proceedings of IEEE 1989 International 
Conference on Systems Engineering, pp. 557-560, OH, USA, 1989.

[19] M. O. Tokhi and A. K. M. Azad, "Real-time finite difference simulation of a single-link flexible manipulator system incorporating hub inertia and payload," Proceedings of the Institution of Mechanical Engineers, Part I: Journal of Systems and Control Engineering, vol. 209, no. 19, pp. 21-33, 1995.

[20] M. O. Tokhi, M. A. Hossain, and A. K. M. Azad, "Digital signal processing and parallel processing in the real-time simulation of a flexible manipulator system," Mechanical Systems and Signal Processing, vol. 10, no. 6, pp. 729-745, 1996.

[21] M. O. Tokhi and A. AKM, Flexible Robot Manipulators: Modelling, Simulation and Control, The Institution of Engineering and Technology, London, 2008.

[22] N. Siddque, Intelligent Control: A Hybrid Approach Based on Fuzzy Logic, Neural Networks and Genetic Algorithms, Springer International Publishing, Switzerland, 2014.

[23] J. F. Peza-Solís, G. Silva-Navarro, and N. R. Castro-Linares, "Trajectory tracking control in a single flexible-link robot using finite differences and sliding modes," Journal of Applied Research and Technology, vol. 13, no. 1, pp. 7078, 2015.

[24] H. J. Yang and M. Tan, "Sliding mode control for flexible-link manipulators based on adaptive neural networks," International Journal of Automation and Computing, vol. 15, no. 2, pp. 239-248, 2018.

[25] H. M. Yatim, I. Z. M. Darus, and M. Mohamad, "Parametric Identification and Dynamic Characterisation of Flexible Manipulator System," in Proceedings of the 2012 IEEE Conference on Control, Systems \& Industrial Informatics, pp. 16-21, Bandung, Indonesia, 2012.

[26] M. A. Hossain and M. O. Tokhi, "Real-time design constraints in implementing active vibration control algorithms," International Journal of Automation \& Computing, vol. 3, no. 3, pp. 252-262, 2006.

[27] G. Chen and D. L. Russell, "A mathematical model for linear elastic systems with structural damping," Quarterly of Applied Mathematics, vol. 39, no. 4, pp. 433-454, 1980.

[28] I. Lasiecka and R. Triggiani, Control Theory for Partial Differential Equations: Continuous and Approximation Theories, Cambridge University Press, New York, 2000.

[29] F. Matsuno, T. Ohno, and Y. V. Orlov, "Proportional derivative and strain (PDS) boundary feedback control of a flexible space structure with a closed-loop chain mechanism," Automatica, vol. 38, no. 7, pp. 1201-1211, 2002.

[30] D. Sun, J. K. Mills, J. J. Shan, and S. K. Tso, “A PZT actuator control of a single-link flexible manipulator based on linear velocity feedback and actuator placement," Mechatronics, vol. 14, no. 4, pp. 381-401, 2004.

[31] F. S. Liu, D. P. Jin, and H. Wen, "Optimal vibration control of curved beams using distributed parameter models," Journal of Sound and Vibration, vol. 384, pp. 15-27, 2016.

[32] J. C. Strikwerda, Finite Difference Schemes and Partial Differential Equations, Society for Industrial and Applied Mathematics, Philadelphia, 2nd edition, 2004.

[33] Z. Yang, X. Chen, X. Li, Y. Jiang, H. Miao, and Z. He, "Wave motion analysis in arch structures via wavelet finite element method," Journal of Sound and Vibration, vol. 333, no. 2, pp. 446-469, 2014.
[34] W. He, T. Wang, X. He, L. J. Yang, and O. Kaynak, "Dynamical modeling and boundary vibration control of a rigid-flexible wing system," IEEE/ASME Transactions on Mechatronics, vol. 25, no. 6, pp. 2711-2721, 2020.

[35] W. He, T. Meng, X. He, and C. Sun, "Iterative learning control for a flapping wing micro aerial vehicle under distributed disturbances," IEEE Transactions on Cybernetics, vol. 49, no. 4, pp. 1524-1535, 2019. 